\title{
Giant photonic spin Hall effect in momentum space in a structured metamaterial with spatially varying birefringence
}

\author{
Xiaohui Ling ${ }^{1,2,3}$, Xinxing Zhou ${ }^{1}$, Xunong $\mathrm{Yi}^{3}$, Weixing Shu ${ }^{1}$, Yachao Liu ${ }^{1}$, Shizhen Chen ${ }^{1}$, Hailu Luo ${ }^{1}$, \\ Shuangchun Wen ${ }^{1}$ and Dianyuan Fan ${ }^{1,3}$
}

The photonic spin Hall effect (SHE) in the reflection and refraction at an interface is very weak because of the weak spin-orbit interaction. Here, we report the observation of a giant photonic SHE in a dielectric-based metamaterial. The metamaterial is structured to create a coordinate-dependent, geometric Pancharatnam-Berry phase that results in an SHE with a spin-dependent splitting in momentum space. It is unlike the SHE that occurs in real space in the reflection and refraction at an interface, which results from the momentum-dependent gradient of the geometric Rytov-Vladimirskii-Berry phase. We theorize a unified description of the photonic SHE based on the two types of geometric phase gradient, and we experimentally measure the giant spin-dependent shift of the beam centroid produced by the metamaterial at a visible wavelength. Our results suggest that the structured metamaterial offers a potential method of manipulating spin-polarized photons and the orbital angular momentum of light and thus enables applications in spin-controlled nanophotonics.

Light: Science \& Applications (2015) 4, e290; doi:10.1038/Isa.2015.63; published online 22 May 2015

Keywords: geometric phase; metamaterial; photonic spin Hall effect

\section{INTRODUCTION}

Metamaterials or metasurfaces are artificial materials that are engineered to produce nearly any imaginable optical properties that are not found in nature. ${ }^{1,2}$ They are typically structured at the subwavelength scale with ultrathin metallic or dielectric micro/nanoparticles or with holes opened in metallic films. Metamaterials exhibit unprecedented degrees of freedom in the polarization and phase manipulation of light via the geometric structuring of their structural units, especially on the wavelength scale, ${ }^{3-9}$ which leads to applications such as vortex beam generators, ${ }^{3,7}$ metalenses ${ }^{10,11}$ and optical holography. ${ }^{12,13}$ These materials also offer considerable potential for the manipulation of the angular moment of light and the photonic spin Hall effect (SHE), thereby providing convenient opportunities for spin-polarized photonics and nanophotonics. ${ }^{14-17}$

The photonic SHE describes the mutual influence of the photon spin (polarization) and the trajectory (orbital angular momentum) of light-beam propagation, i.e., the spin-orbit interaction (SOI), which results in two types of geometric phases: the Rytov-VladimirskiiBerry (RVB) phase and the Pancharatnam-Berry (PB) phase. ${ }^{18-22}$ The RVB phase is associated with the evolution of the propagation direction of light. When a light beam reflects/refracts at a planar interface between different media, a SOI occurs, and the corresponding momentum-dependent RVB phase leads to a spin-dependent real- space (coordinate) shift, i.e., the photonic SHE. ${ }^{19,22,23}$ However, this SOI is generally very weak, and the induced spin-dependent shift is also exceedingly tiny (subwavelength scale), with few exceptions. ${ }^{24}$ The detection of this tiny shift requires weak measurement technology or multiple reflections. ${ }^{20,22}$ Thus, controlling and, in particular, enhancing the SHE are important for directly exploiting the spin and the orbital angular momentum of light for information processing, quantum computing and communication, and it thus provides a route to spin-controlled photonics. ${ }^{15-17}$ If one employs the rapidly varying dynamic phase discontinuities produced by metallic plasmonic antennas at the medium interface, it is possible to observe an enhanced SOI and a giant photonic SHE. ${ }^{16}$

Moreover, a photonic SHE related to another geometric phase, the PB phase, has also been reported. ${ }^{14,17,25}$ The PB phase is related to a change in the polarization of light. In certain inhomogeneous anisotropic media, a spatially varying $\mathrm{PB}$ phase has been produced to generate and manipulate vortex beams, vector beams, vector vortex beams, etc. ${ }^{26-31}$ In this work, we theorize a unified description of the photonic SHE caused by the two types of geometric phases. It is predicted that the spin-dependent shift induced by the PB phase gradient can be very large because it occurs in $k$ (momentum) space. Upon beam propagation, the $k$-space shift induces a real-space shift that may be much larger than that produced by the RVB phase. To verify this

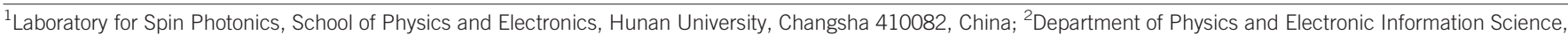

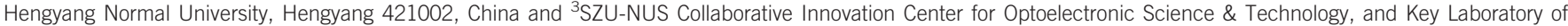
Optoelectronic Devices and Systems of Ministry of Education and Guangdong Province, Shenzhen University, Shenzhen 518060, China Correspondence: HL Luo, Laboratory for Spin Photonics, School of Physics and Electronics, Hunan University, Changsha 410082, China 
prediction, a dielectric-based metamaterial at a visible wavelength is structured to create a PB phase gradient in one dimension, which leads to the observation of a giant photonic SHE. Note that a polarizationdependent beam splitting and focusing effect in subwavelength diffraction gratings associated with the $\mathrm{PB}$ phase has been reported previously, but it was limited to the infrared spectral domain. ${ }^{32,33}$ Recently, ultrathin gratings, lenses and axicons in the optical range have also been realized by means of dielectric gradient metasurfaces. ${ }^{34}$ They are fabricated by patterning a semiconductor film with the desired nanostructures, and their operational mechanism is attributed to polarization diffraction. In fact, they can be well described by the theory presented here.

\section{MATERIALS AND METHODS}

Unified description of the photonic SHE based on the geometric phase gradient

Let us first provide a brief review of the photonic SHE associated with the RVB phase occurring in the reflection and refraction of a light beam at an interface between different media and propose a unified description for the two types of photonic SHE based on the geometric phase gradient. Because of the change in the beam propagation direction, the angular spectral components of the beam with different wave vectors acquire different RVB phases, ${ }^{20,22}$ thus creating a geometric phase gradient along the interface. This phase gradient manifests as a spin-dependent shift $\Delta r=r_{x}+r_{y}$ in real space. It can be described by the following expression:

$$
\Delta r=\nabla \Phi_{G}\left(k_{x}, k_{y}\right)
$$

where $\Phi_{G}\left(k_{x}, k_{y}\right)$ is the geometric RVB phase. It is a function of the transverse wave vector of the light. Assume that $z$ is the normal direction and that $(x, z)$ is the plane of incidence. Thus, $k_{x}$ and $k_{y}$ are the in-plane (incidence plane) and out-of-plane wave vectors, respectively. The $k_{x}$-dependent RVB phase is responsible for the in-plane spin separation, ${ }^{35,36}$ whereas the $k_{y}$-dependent RVB phase will result in a spin-dependent shift perpendicular to the incidence plane, i.e., the SHE of the light. ${ }^{18,19,22}$

Here, we are interested only in the SHE, that is, we consider $\Phi_{\mathrm{G}}$ to be dependent only on $k_{y}$. For typical interfacial reflection and refraction, the RVB phase can be given as ${ }^{22,37}$

$$
\boldsymbol{\Phi}_{G}\left(k_{y}\right)=\sigma_{ \pm} k_{y} \delta
$$

with $\sigma_{+}=+1$ and $\sigma_{-}=-1$ indicating left- and right-handed circular polarizations, respectively. $\delta \propto \cot \theta_{i} / k_{0}$ is related to the optical parameters (thickness, refractive indices, etc.) of the interface, with $\theta_{i}$ being the incident angle and $k_{0}=2 \pi / \lambda$ being the vacuum wave vector. ${ }^{22,37}$ The photonic SHE can be tuned by modulating the optical parameters of the interface. ${ }^{37}$ In turn, the SHE also provides an advantageous metrology for measuring the optical parameters of nanostructures such as metallic films and graphene. ${ }^{38,39}$ Note that Equation (2) also indicates the SOI between the spin $\sigma_{ \pm}$and the transverse momentum $k_{y}$ of the beam.

Now, we can calculate the spin-dependent shift of the beam centroid as the gradient of $\Phi_{G}\left(k_{x}, k_{y}\right)$ :

$$
\Delta r_{y}=\nabla \boldsymbol{\Phi}_{G}\left(k_{y}\right)=\frac{\partial \boldsymbol{\Phi}_{G}}{\partial k_{x}} \hat{e}_{x}+\frac{\partial \boldsymbol{\Phi}_{G}}{\partial k_{y}} \hat{e}_{y}=\sigma_{ \pm} \delta \hat{e}_{y}
$$

where $\hat{e}_{x}\left(\hat{e}_{y}\right)$ is the unit vector in the $x(y)$ direction. In this case, $\Delta r_{y}$ is a constant with a magnitude equal to $\delta$, which is a small quantity limited by a fraction of $\lambda$.
We then generalize this discussion to the geometric PB phase. The $\mathrm{PB}$ phase is related to the change in the polarization of light. Typically, when a light beam normally passes through a birefringent waveplate, an additional phase that is dependent only on the optical axis direction of the waveplate is introduced, i.e., the PB phase. ${ }^{40}$ It is expected that the light beam will acquire a space-variant $\mathrm{PB}$ phase when propagating through an inhomogeneous anisotropic medium with homogeneous phase retardation, such as an inhomogeneous subwavelength grating, a liquid crystal $q$-plate, or a plasmonic metasurface. ${ }^{26-28}$ The PB phase can be written as $\Phi_{G}(x, y)=-2 \sigma_{ \pm} \alpha(x, y)$, where $\alpha(x, y)$ is the local optical axis direction of the anisotropic medium. The factor -2 arises because the anisotropic medium reverses the handedness of the circular polarization and applies an additional geometric phase factor $2 \sigma_{ \pm} \alpha(x, y)$ to the output light. The Jones matrix transformation of this process, irrespective of loss, is expressed as

$$
\left(\begin{array}{l}
1 \\
\sigma_{ \pm} i
\end{array}\right) \rightarrow \cos \frac{\psi}{2}\left(\begin{array}{l}
1 \\
\sigma_{ \pm} i
\end{array}\right)+i \sin \frac{\psi}{2}\left(\begin{array}{l}
1 \\
\sigma_{\mp} i
\end{array}\right) e^{i 2 \sigma_{ \pm} \alpha(x, y)}
$$

where $\psi$ is the phase retardation of the medium. The above equation indicates that a fraction $\sin ^{2}(\psi / 2)$ of the incident photons reverse their handedness and that the remaining photon fraction $\cos ^{2}(\psi / 2)$ remains unchanged. Thus, $\psi$ determines the conversion efficiency. For $\psi=\pi$, i.e., a half-wave phase retardation, all photons reverse their handedness (see the schematic illustrations in Figure 1).

The coordinate-dependent $\mathrm{PB}$ phase creates a geometric phase gradient along the medium surface,
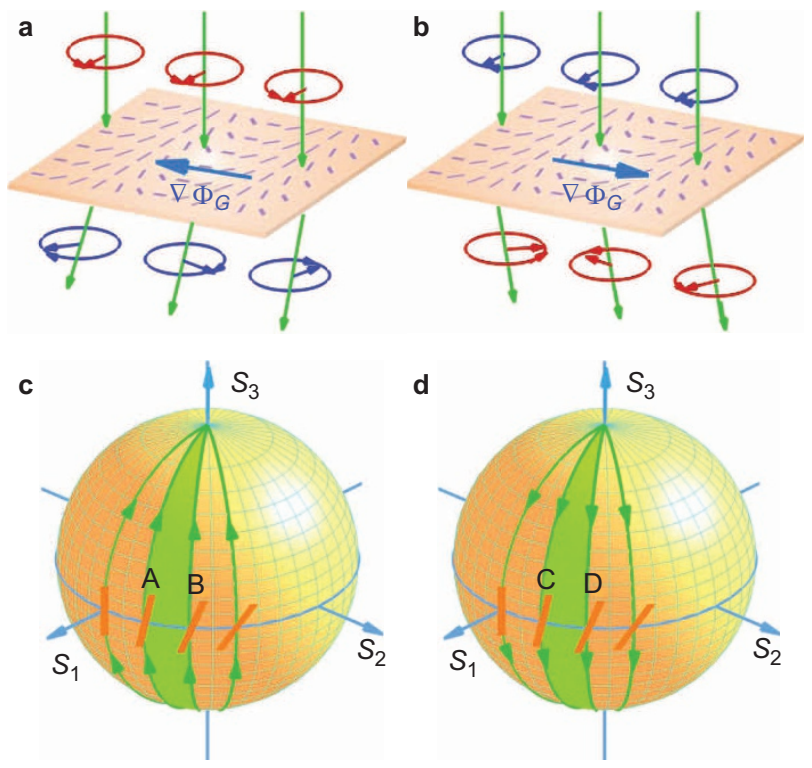

Figure 1 Schematic illustration of the photonic SHE in a structured metamaterial and the corresponding polarization evolution on the Poincaré sphere. (a) and (b) illustrate the conversion of spin states upon the transmission of circularly polarized light in a structured metamaterial with a spin-dependent PB phase gradient $\nabla \Phi_{G}$. The short lines indicate the local optical axis direction of the metamaterial. For $\sigma_{+}$and $\sigma_{-}$incidence, the metamaterial simply produces an opposite $\nabla \Phi_{G}$ and an opposite spin-dependent momentum shift (deflection of optical rays). (c) and (d) illustrate the evolution of the polarization states on the Poincaré sphere. The trajectories (A, B) and (C, D) represent the conversions of the initial spin states in metamaterials with different local optical axes, corresponding to (a) and (b), respectively. The half of the solid angles encompassed by the green areas indicate the corresponding PB phases. ${ }^{26} \mathrm{~PB}$, Pancharatnam-Berry; SHE, spin Hall effect. 


$$
\Delta k(x, y)=\nabla \boldsymbol{\Phi}_{G}(x, y)=\frac{\partial \mathbf{\Phi}_{G}}{\partial x} \hat{e}_{x}+\frac{\partial \mathbf{\Phi}_{G}}{\partial y} \hat{e}_{y}
$$

This shift occurs in $k$ space. Here, for simplicity, we design a structured anisotropic metamaterial with homogeneous phase retardation such that its optical axis direction is periodically varying in one dimension, e.g., $\alpha(x, y)=\Omega x$, where $\Omega=\pi / d$ is the spatial rotational rate of the optical axis, with $d$ being the period.

For circularly polarized light passing through the metamaterial, its handedness reverses and acquires an additional spatially varying phase, i.e., the $\mathrm{PB}$ phase. This is graphically illustrated by the Poincaré sphere, where $\sigma_{ \pm}$light evolves into $\sigma_{\mp}$ light from the north (south) pole to the south (north) pole along different paths (Figure 1c and 1d). In this case, the inhomogeneous PB phase creates a phase gradient, and the spin-dependent momentum shift occurs only in the $x$ direction, i.e.,

$$
\Delta k_{x}=-2 \sigma_{ \pm} \Omega=-2(\pi / d) \hat{e}_{x}
$$

In fact, it is an angular shift, and the induced real-space shift increases linearly during beam propagation. According to the mapping relationship between momentum space and real space (Figure $2 \mathrm{~d}$ ), we find that

$$
\Delta x=\left(\Delta k / k_{0}\right) z=-\sigma_{ \pm} \lambda z / d
$$

Hence, from the observed $\Delta x$, we can directly obtain $\Delta k$.

\section{Fabrication of the metamaterial sample}

To demonstrate the PB phase and the induced spin-dependent splitting, we designed an inhomogeneous metamaterial with a periodically varying optical axis orientation in one dimension. When a light beam

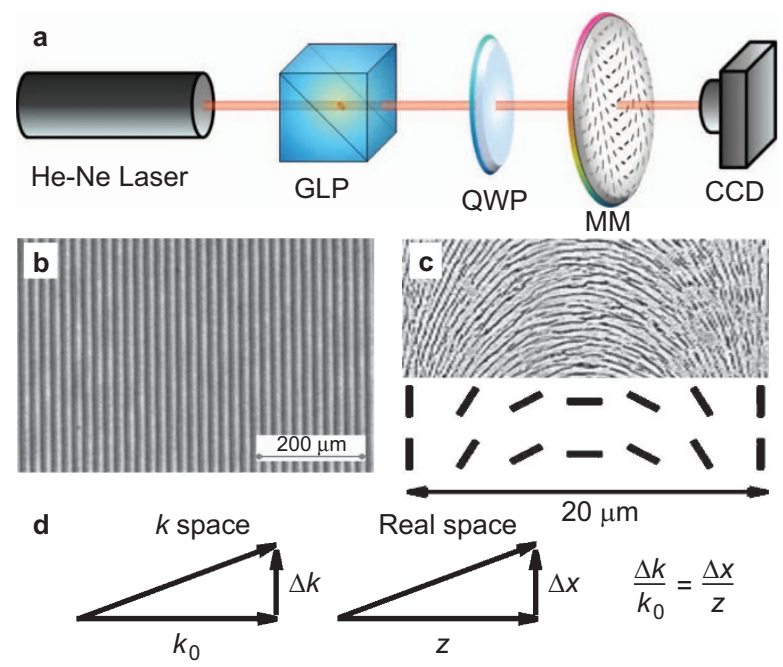

Figure 2 Experimental demonstration of the PB phase gradient and the giant photonic SHE. (a) Experimental apparatus. A He-Ne laser produces a linearly polarized Gaussian beam. A GLP and a QWP can modify the beam into any desired elliptically polarized beam. Then, the beam passes through a structured metamaterial (MM), and the output intensity can be recorded by a CCD or imaged on an observation plane. (b) shows a photograph of the metamaterial. (c) The detailed geometry of the metamaterial over one period $(20 \mu \mathrm{m})$ and a schematic illustration of the local optical axis (slow axis). (d) The mapping relationship between the momentum shift $\Delta k$ and the induced real-space shift $\Delta x$. CCD, charge-coupled device; GLP, Glan laser polarizer; PB, Pancharatnam-Berry; QWP, quarter-wave plate; SHE, spin Hall effect. passes through this metamaterial, a PB phase gradient that is dependent upon the spatial rotational rate $\Omega$ of the local optical axis is created. By suitably engineering $\Omega$, we can obtain a metamaterial with either a positive or negative PB phase gradient. Such a metamaterial can be fabricated via the femtosecond laser writing of spatially varying nanogrooves in a fused silica sample. The laser beam is focused $200 \mu \mathrm{m}$ below the surface of the glass sample. ${ }^{41}$ Under intense laser irradiation, the uniform glass $\left(\mathrm{SiO}_{2}\right)$ decomposes into porous glass $\left(\mathrm{SiO}_{2(1-x)}+x \mathrm{O}_{2}\right)$, whose refractive index depends upon the laser intensity. ${ }^{42}$ Thus, a periodic change in the intensity can lead to a modulation of the refractive index, i.e., can generate grating-like nanostructures, and result in the formation of birefringence in the isotropic glass sample. The local optical axis directions (fast and slow axes) are perpendicular and parallel to the grooves, respectively. ${ }^{43}$ Because the characteristic dimension of the structure is much smaller than the operational wavelength, the fabricated metamaterial can be regarded as a birefringent waveplate with homogeneous phase retardation and a locally varying optical axis direction. The phase retardation is $\psi=2 \pi\left(n_{e}-n_{o}\right) h / \lambda$, where $h$ is the writing depth and $n_{e^{-}} n_{o}$ is the induced birefringence. As a linear approximation, the effective ordinary and extraordinary refractive indices can be written as ${ }^{43}$

$$
n_{o}=\sqrt{f n_{1}^{2}+(1-f) n_{2}^{2}}, \quad n_{e}=\sqrt{\frac{n_{1}^{2} n_{2}^{2}}{f n_{2}^{2}+(1-f) n_{1}^{2}}}
$$

Here, $f$ is the duty cycle, and $n_{1}$ and $n_{2}$ are the refractive indices of the two media that form the grating-like structure of the metamaterial. We entrusted the sample fabrication to Altechna Co. Ltd. The fabrication method is described in Refs. 41 and 43. Two metamaterials with $\Omega= \pm \pi / 20 \mathrm{rad} \mu \mathrm{m}^{-1}$ were prepared. The dimension of each sample is $6 \mathrm{~mm} \times 6 \mathrm{~mm}$. At a wavelength of $633 \mathrm{~nm}$, the writing depth is approximately $80 \mu \mathrm{m}$, the line width is $30-50 \mathrm{~nm}$, the duty cycle is $0.1-0.2$, the writing period is $250-300 \mathrm{~nm}$ and the value of $n_{e}-n_{o}$ is estimated to be $-(2-4) \times 10^{3}$. Figure $2 \mathrm{~b}$ presents a photograph of the metamaterial. One period of the metamaterial is depicted in Figure 2c.

\section{RESULTS AND DISCUSSION}

Experimental demonstration of the $\mathrm{PB}$ phase gradient and the spin-dependent splitting in momentum space

The experimental apparatus is depicted in Figure 2. An He-Ne laser (wavelength $\lambda=633 \mathrm{~nm}$ ) outputs a linearly polarized fundamentalmode Gaussian beam. A Glan laser polarizer and a quarter-wave plate are employed to modify the polarization state. Then, the laser beam passes through the structured metamaterial, and the output intensity is recorded by a CCD camera. Because the $\Delta k$-induced shift increases linearly with the transmission distance $z$, we experimentally measure the shifts at different observation planes far from the metamaterial and plot them in Figure 3. We designed the metamaterial with a period of $d=20 \mu \mathrm{m}$; therefore, the rotational rate is $\Omega=\pi / 20 \mathrm{rad} \mu \mathrm{m}^{-1}$. According to Equation (7), the $k$-induced shift upon transmission can be calculated as $\Delta x=-\sigma_{ \pm} 0.03165 z$. This agrees well with the measured results. We also designed another metamaterial with precisely the opposite rotational rate $\left(\Omega=-\pi / 20 \mathrm{rad} \mu \mathrm{m}^{-1}\right)$ with respect to the previous one; in this case, of course, the direction of the spin accumulation is reversed.

We record the output intensity at a distance of $10 \mathrm{~cm}$ away from the metamaterial for a linearly polarized incident beam, as shown in Figure 4 . The intensity profile exhibits three spots. The central spot retains the same linear polarization as that of the incident beam, with 

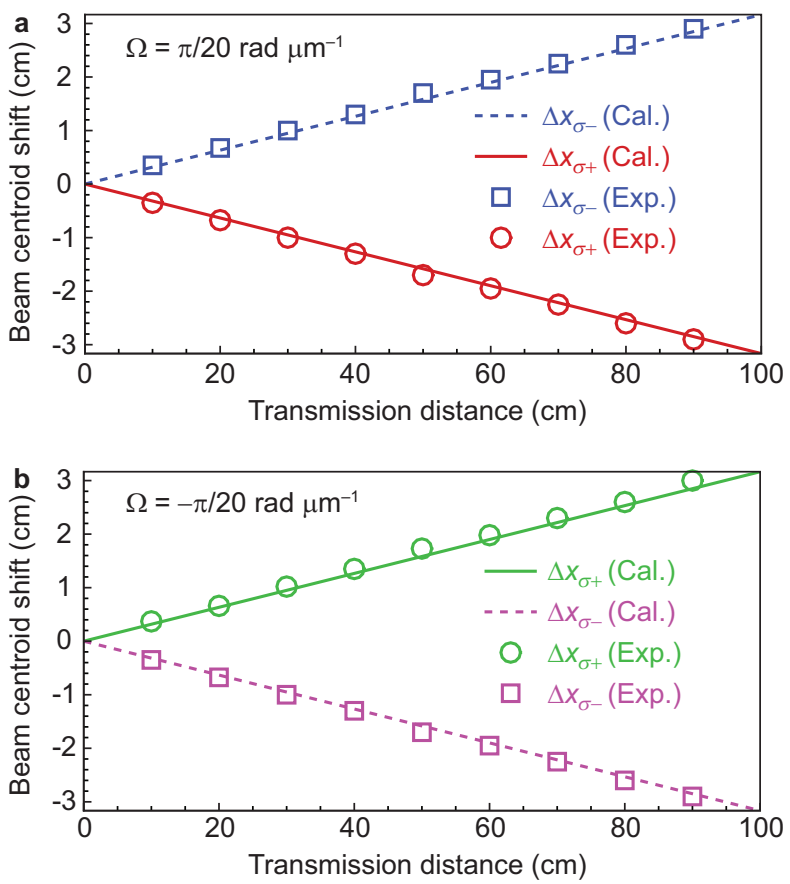

Figure 3 Spin-dependent real-space shift induced by the momentum shift during beam propagation. (a) The calculated (Cal.) and experimental (Exp.) results

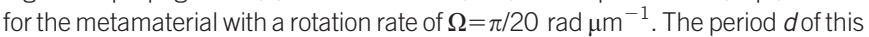
metamaterial sample is $20 \mu \mathrm{m}$. (b) The corresponding results for another sample with the exactly opposite rotational rate of $\Omega=-\pi / 20 \mathrm{rad} \mu \mathrm{m}^{-1}$.

an intensity much lower than those of the other two spots. This spot represents the unconverted portion of the incident photons (Equation (4)). This means that the metamaterial does not produce exact $\pi$ retardation. The phase retardation depends upon the thickness of the metamaterial; however, the real thickness of the sample exhibits
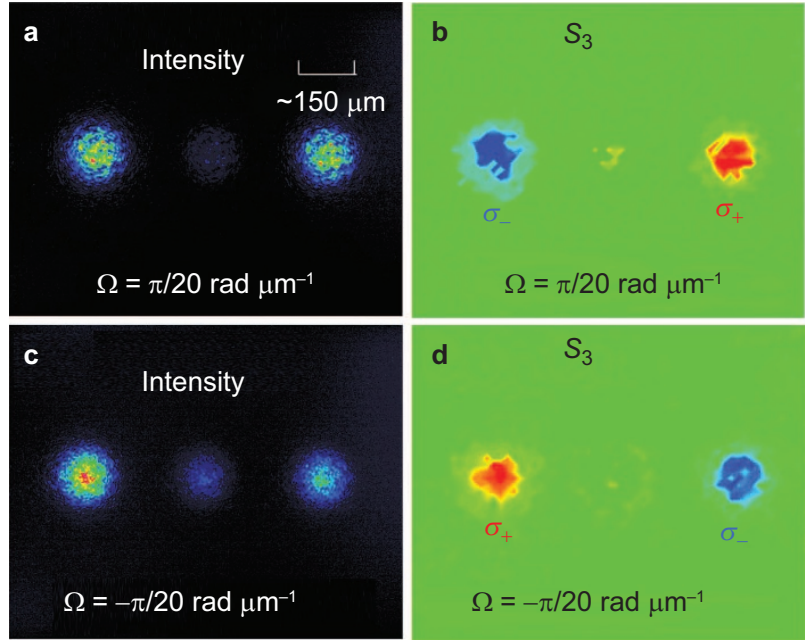

Figure 4 Intensities recorded by the CCD and the corresponding $S_{3}$ parameters after a linearly polarized beam passes through two structured metamaterials with exactly opposite rotational rates. The observation plane is located $10 \mathrm{~cm}$ away from the metamaterial. (a) and (b) show the intensity and $S_{3}$ parameter for the metamaterial with a rotation rate of $\Omega=\pi / 20 \mathrm{rad}_{\mu \mathrm{m}^{-1}}$, respectively. (c) and (d) are the corresponding results for another sample with the exactly opposite rotational rate of $\Omega=-\pi / 20$ rad $\mu \mathrm{m}^{-1}$. CCD, charge-coupled device. a slight deviation from the desired value. The left and right spots represent the $\sigma_{+}$and $\sigma_{-}$components, respectively, which can be discriminated by measuring the Stokes parameter $S_{3}$ because the $S_{3}$ parameter can be used to describe the degree of circular polarization. Note that for $\Omega=-\pi / 20 \mathrm{rad} \mu \mathrm{m}^{-1}$, the direction of the spin accumulation is simply reversed.

When an elliptically polarized light beam passes through the metamaterial, it is expected that a non-symmetric spin-dependent splitting of the light will be observed. Because an elliptically polarized light beam can be regarded as a superposition of two circular polarizations with unequal magnitudes, the spin-dependent splitting manifests as an asymmetric intensity splitting. The two spin components still exhibit the same shifts because the $\mathrm{PB}$ phase is dependent only on the spatial rotational rate of the metamaterial. We choose several typical ellipticities of the incident polarization and plot the experimental results in Figure 5. Note that the central spot represents the unconverted portion of the incident beam.

Transmission and conversion efficiency of the metamaterial sample The transmittance of the metamaterial sample at a wavelength of $633 \mathrm{~nm}$ is measured using a laser power meter. Let us consider the metamaterial with $\Omega=\pi / 20 \mathrm{rad} \mu \mathrm{m}^{-1}$. The transmittance is approximately $50.1 \%$. This is the average value from four sets of data measured at four different locations on the metamaterial. The energy losses are attributed to microscopic inhomogeneities and induced defect absorption, which may be reduced by optimizing the fabrication process. $^{41}$

The conversion efficiency is also measured. It is dependent on the phase retardation of the metamaterial. For a writing depth of $h=\lambda /$ $2\left(n_{e}-n_{o}\right)$, the phase retardation $\psi$ is equal to $\pi$. From Equation (4), we know that the theoretical conversion efficiency of the metamaterial is $100 \%$. It can be estimated from Figure 4 a that the actual conversion efficiency approaches this ideal value. We also implement several sets of experiments to evaluate the conversion efficiency, which is found to be approximately $96.3 \%$ for the metamaterial with $\Omega=\pi / 20 \mathrm{rad}$ $\mu \mathrm{m}^{-1}$. For the metamaterial with $\Omega=-\pi / 20 \mathrm{rad} \mu \mathrm{m}^{-1}$, the measured conversion efficiency is approximately $75.5 \%$ which deviates considerably from the theoretical prediction. This deviation can be attributed to a lack of precision in the writing depth or the birefringence. Note that the losses are not considered when evaluating the conversion efficiency from the measured data.

\section{Discussion}

In our scenario, the $\mathrm{PB}$ phase gradient induces a constant momentum shift, i.e., a spin-dependent angular deflection. The observable quantity is the real-space shift that is induced by the momentum shift during beam propagation, which increases linearly with the propagation distance $z$. A larger rotation rate of the metamaterial leads to a more rapid increase in the induced shift. A negative rotation rate will reverse the direction of spin accumulation. The rotation rate can be tuned by modulating the structural geometry of the metamaterial. The photonic SHE that occurs in the interfacial reflection and refraction exhibits a constant beam shift during beam propagation, which is limited to a fraction of the wavelength. ${ }^{19,22-24}$ The detection of such a tiny shift in the visible range requires weak measurement technology. By contrast, the spin-dependent shift induced by the metamaterial here is sufficiently large to allow for direct measurement. Therefore, such a structured metamaterial offers an additional degree of freedom for the manipulation of the photonic SHE, especially in the effort to obtain a giant photonic SHE. 

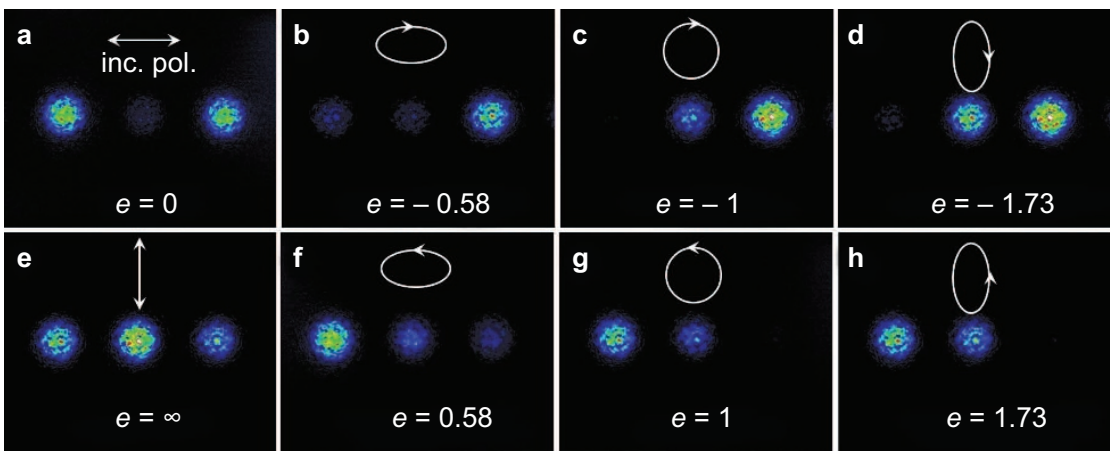

Figure 5 Asymmetric spin-dependent splitting of the metamaterial $\left(\Omega=\pi / 20 \mathrm{rad}_{\mu} \mathrm{m}^{-1}\right)$ for incident beams with different elliptical polarizations. e represents the ellipticity of the incident polarization (inc. pol.) ellipse. The observation plane is located $10 \mathrm{~cm}$ away from the metamaterial. (a-h) Intensities recorded by the CCD for incident beams with $e=0,-0.58,-1,-1.73, \infty, 0.58,1$ and 1.73 , respectively. CCD, charge-coupled device.

We also note that in some subwavelength diffraction gratings, a polarization-dependent beam splitting and focusing effect has been observed. ${ }^{32,33}$ The possibility of realizing ultrathin gratings, lenses, and axicons using dielectric gradient metasurfaces has also been demonstrated. $^{34}$ The operational mechanism of these materials is attributed to the polarization diffraction effect; however, it can also be well described by the theory presented here.

\section{CONCLUSIONS}

From the perspective of a geometric phase gradient, we proposed, to our knowledge, the first unified description of the photonic SHEs related to the two types of geometric phase gradients. Dielectric metamaterials were fabricated to experimentally demonstrate the $\mathrm{PB}$ phase gradient and to allow for the observation of a giant photonic SHE in momentum space at a visible wavelength, originating from the locally varying optical axis direction of the metamaterial. Unlike the tiny realspace shift induced by the RVB phase gradient, the shift induced by the $\mathrm{PB}$ phase gradient of the metamaterial occurs in momentum space and is sufficiently large to be observed directly. This approach may bridge the gap between spin-based photonics and nanophotonics and thus provide an opportunity for manipulating the spin and orbital angular momentum of light.

\section{ACKNOWLEDGEMENTS}

This research was partially supported by the National Natural Science Foundation of China (Grants No. 11274106, No. 11474089 and No. 11447010), the China Postdoctoral Science Foundation (Grant No. 2014M562198), the Scientific Research Fund of Hunan Provincial Education Department of China (Grant No. 13B003) and the Natural Science Foundation of Hunan Province (Grant No. 2015JJ3026).

1 Kildishev AV, Boltasseva A, Shalaev VM. Planar photonics with metasurfaces. Science 2013; 339: 1232009.

2 Yu NF, Capasso F. Flat optics with designer metasurfaces. Nat Mater 2014; 13: 139150.

3 Yu NF, Genevet P, Kats MA, Aieta F, Tetienne JP et al. Light propagation with phase discontinuities: generalized laws of reflection and refraction. Science 2011; 334: 333-337.

4 Zhao Y, Alù A. Manipulating light polarization with ultrathin plasmonic metasurfaces. Phys Rev B 2011; 84: 205428.

5 Zhao Y, Belkin MA, Alù A. Twisted optical metamaterials for planarized ultrathin broadband circular polarizers. Nat Commun 2012; 3: 870.

6 Aieta F, Genevet P, Yu NF, Kats MA, Gaburro Z et al. Out-of-plane reflection and refraction of light by anisotropic optical antenna metasurfaces with phase discontinuities. Nano Lett 2012; 12: 1702-1706.

7 Genevet P, Yu NF, Aieta F, Lin J, Kats MA et al. Ultra-thin plasmonic optical vortex plate based on phase discontinuities. Appl Phys Lett 2012; 100: 013101.
8 Pors A, Nielsen MG, Eriksen RL, Bozhevolnyi SI. Broadband focusing flat mirrors based on plasmonic gradient metasurfaces. Nano Lett 2013; 13: 829-834.

9 Huang LL, Chen XZ, Bai BF, Tan QF, Jin GF et al. Helicity dependent directional surface plasmon polariton excitation using a metasurface with interfacial phase discontinuity. Light Sci App/2013; 2: e70; doi:10.1038/Isa.2013.26.

10 Chen XZ, Huang LL, Mühlenbernd H, Li GX, Bai BF et al. Dual-polarity plasmonic metalens for visible light. Nat Commun 2012; 3: 1198.

$11 \mathrm{Ni}$ XJ, Ishii S, Kildishev AV, Shalaev VM. Ultra-thin, planar, Babinet-inverted plasmonic metalenses. Light Sci Appl 2013; 2: e72; doi:10.1038/Isa.2013.28.

$12 \mathrm{NiXJ}$, Kildishev AV, Shalaev VM. Metasurface holograms for visible light. Nat Commun 2013; 4: 2807.

13 Huang LL, Chen XZ, Mühlenbernd H, Zhang H, Chen SM et al. Three-dimensional optical holography using a plasmonic metasurface. Nat Commun 2013; 4: 2808.

14 Shitrit N, Bretner I, Gorodetski Y, Kleiner V, Hasman E. Optical spin Hall effects in plasmonic chains. Nano Lett 2011; 11: 2038-2042.

15 Shitrit N, Yulevich I, Maguid E, Ozeri D, Veksler D et al. Spin-optical metamaterial route to spin-controlled photonics. Science 2013; 340: 724-726.

16 Yin XB, Ye ZL, Rho J, Wang Y, Zhang X. Photonic spin Hall effect at metasurfaces. Science 2013; 339: 1405-1407.

17 Li GX, Kang M, Chen S, Zhang S, Pun EYB et al. Spin-enabled plasmonic metasurfaces for manipulating orbital angular momentum of light. Nano Lett 2013; 13: 41484151.

18 Onoda M, Murakami S, Nagaosa N. Hall effect of light. Phys Rev Lett 2004; 93 : 083901.

19 Bliokh KY, Bliokh YP. Conservation of angular momentum, transverse shift, and spin Hall effect in reflection and refraction of an electromagnetic wave packet. Phys Rev Lett 2006; 96: 073903.

20 Bliokh KY, Niv A, Kleiner V, Hasman E. Geometrodynamics of spinning light. Nat Photonics 2008; 2: 748-753.

21 Bliokh KY, Gorodetski Y, Kleiner V, Hasman E. Coriolis effect in optics: unified geometric phase and spin-Hall effect. Phys Rev Lett 2008; 101: 030404.

22 Hosten 0, Kwiat P. Observation of the spin Hall effect of light via weak measurements. Science 2008; 319: 787-790.

23 Qin Y, Li Y, He HY, Gong QH. Measurement of spin Hall effect of reflected light. Opt Lett 2009; 34: 2551-2553.

24 Luo HL, Zhou XX, Shu WX, Wen SC, Fan DY. Enhanced and switchable spin Hall effect of light near the Brewster angle on reflection. Phys Rev A 2011; 84: 043806.

25 Ling XH, Zhou XX, Shu WX, Luo HL, Wen SC. Realization of tunable photonic spin Hall effect by tailoring the Pancharatnam-Berry phase. Sci Rep 2014; 4: 5557.

26 Biener G, Niv A, Kleiner V, Hasman E. Formation of helical beams by use of Pancharatnam-Berry phase optical elements. Opt Lett 2002; 27: 1875-1877.

27 Marrucci L, Manzo C, Paparo D. Optical spin-to-orbital angular momentum conversion in inhomogeneous anisotropic media. Phys Rev Lett 2006; 96: 163905.

28 Karimi E, Schulz SA, de Leon I, Qassim I, Upham J et al. Generating optical orbital angular momentum at visible wavelengths using a plasmonic metasurface. Light Sci App/2014; 3: e167; doi:10.1038/Isa.2014.48.

29 Liu YC, Ling XH, Yi XN, Zhou XX, Luo HL et al. Realization of polarization evolution on higher-order Poincaré sphere with metasurface. App/ Phys Lett 2014; 104: 191110.

30 Bouchard F, De Leon I, Schulz SA, Upham J, Karimi E et al. Optical spin-to-orbital angular momentum conversion in ultra-thin metasurfaces with arbitrary topological charges. Appl Phys Lett 2014; 105: 101905.

31 Yi XN, Ling XH, Zhang ZY, Li Y, Zhou XX et al. Generation of cylindrical vector vortex beams by two cascaded metasurfaces. Opt Express 2014; 22: 17207-17215.

32 Hasman E, Bomzon Z, Niv A, Biener G, Kleiner V. Polarization beam-splitters and optical switches based on space-variant computer-generated subwavelength quasiperiodic structures. Opt Commun 2002; 209: 45-54.

33 Hasman E, Kleiner V, Biener G, Niv A. Polarization dependent focusing lens by use of quantized Pancharatnam-Berry phase diffractive optics. App/ Phys Lett 2003; 82: 328-330.

34 Lin DM, Fan PY, Hasman E, Brongersma ML. Dielectric gradient metasurface optical elements. Science 2014; 345: 298-302. 
35 Qin Y, Li Y, Feng XB, Xiao YF, Yang $\mathrm{H}$ et al. Observation of the in-plane spin separation of light. Opt Express 2011; 19: 9636-9645.

36 Pan MM, Li Y, Ren JL, Wang B, Xiao YF et al. Impact of in-plane spread of wave vectors on spin Hall effect of light around Brewster's angle. Appl Phys Lett 2013; 103: 071106.

37 Luo HL, Ling XH, Zhou XX, Shu WX, Wen SC et al. Enhancing or suppressing the spin Hall effect of light in layered nanostructures. Phys Rev A 2011; 84: 033801.

38 Zhou XX, Ling XH, Luo HL, Wen SC. Identifying graphene layers via spin Hall effect of light. Appl Phys Lett 2012; 101: 251602.

39 Zhou XX, Xiao ZC, Luo HL, Wen SC. Experimental observation of the spin Hall effect of light on a nanometal film via weak measurements. Phys Rev A 2012; 85: 043809.

40 Courtial J. Wave plates and the Pancharatnam phase. Opt Commun 1999; 171: 179183.

41 Beresna M, Gecevièius M, Kazansky PG, Gertus T. Radially polarized optical vortex converter created by femtosecond laser nanostructuring of glass. App Phys Lett 2011; 98: 201101 .
42 Shimotsuma Y, Kazansky PG, Qiu J, Hirao K. Self-organized nanogratings in glass irradiated by ultrashort light pulses. Phys Rev Lett 2003; 91: 247405.

43 Beresna M, Gecevièius M, Kazansky PG. Polarization sensitive elements fabricated by femtosecond laser nanostructuring of glass. Opt Mater Express 2011; 1: 783795.

(c) (i) $(-)$ This work is licensed under a Creative Commons AttributionNonCommercial-NoDerivs 3.0 Unported License. The images or other third party material in this article are included in the article's Creative Commons license, unless indicated otherwise in the credit line; if the material is not included under the Creative

Commons license, users will need to obtain permission from the license holder to reproduce the material. To view a copy of this license, visit http://creativecommons.org/licenses/ by-nc-nd/3.0/ 